\title{
Diffusion-driven GaInP/GaAs light-emitting diodes enhanced by modulation doping
}

\author{
Antti Myllynen ${ }^{1}$ (D) $\cdot$ Toufik Sadi $^{1} \cdot$ Jani Oksanen ${ }^{1}$ \\ Received: 30 November 2018 / Accepted: 5 March 2019 / Published online: 14 March 2019 \\ (c) The Author(s) 2019
}

\begin{abstract}
Diffusion-driven charge transport (DDCT) in III-V light-emitting diodes (LEDs) can enable unconventional optoelectronic devices and functionality by fundamentally changing device design and the current injection principle. In our recent study, an AlGaAs/GaAs DDCT-LED consisting of an array of lateral heterojunctions was studied for large-area applications at high powers. Here, we investigate the current spreading and recombination uniformity of a modulation doped GaInP/GaAs DDCT-LED. In particular, we analyze how the background doping of the lower GaInP cladding layer (CL) and the GaAs substrate changes the carrier distribution within the active region of the device. Our charge transport simulations based on the drift-diffusion current and continuity equations predict that modulation doping by a p-doped CL provides much higher recombination uniformity at high powers compared to an n-doped CL. Most importantly, improved current spreading is achieved while maintaining excellent device performance.
\end{abstract}

Keywords Diffusion-driven charge transport (DDCT) $\cdot$ Light-emitting diode (LED) · Lateral heterojunction $(\mathrm{LHJ}) \cdot$ Current spreading $\cdot$ Modulation doping

\section{Introduction}

Past decades have witnessed extensive research on planar optoelectronic devices based on GaN (Krames et al. 2007c; Nakamura and Krames 2013), and recently also the interest on III-As based light-emitting devices has started to increase again (Olsson et al. 2016; Xiao et al. 2018). However, the performance of high-power light-emitting diodes (LEDs), using traditional designs based on a double heterojunction (DHJ) structure, where electrons and

This article is part of the Topical Collection on Numerical Simulation of Optoelectronic Devices, NUSOD' 18.

Guest edited by Paolo Bardella, Weida Hu, Slawomir Sujecki, Stefan Schulz, Silvano Donati, Angela Traenhardt.

Antti Myllynen

antti.i.myllynen@aalto.fi

1 Engineered Nanosystems Group, Department of Neuroscience and Biomedical Engineering, Aalto University, P.O. Box 12200, 00076 Aalto, Finland 
holes are injected into the active region (AR) from opposite sides, is still limited by inefficient current spreading at high bias voltages. This leads to current crowding and suboptimal device performance due to the highly concentrated light emission close to the p-contact edge.

The diffusion-driven charge transport (DDCT) injection method (Kivisaari et al. 2013; Riuttanen et al. 2014, 2015a, b) was recently proposed to reduce the limitations of conventional GaN LEDs, including current crowding, lateral resistance (Kivisaari et al. 2017c) and resistive losses (Kivisaari et al. 2017a). The DDCT injection is based on bipolar electron and hole diffusion currents that are used to inject charge carriers into ARs located outside the $\mathrm{p}-\mathrm{n}$ junction. This was suggested to enable new device designs where singlesided electrical injection of lateral heterojunctions (LHJs) increases effective device area and removes the need of light-blocking top contacts, if the light is extracted from the bottom side of the device.

Recently, we suggested that a laterally doped AlGaAs/GaAs DDCT-LED structure (Myllynen et al. 2018) can eliminate the non-radiative surface recombination that can be quite detrimental for high efficiency III-As LEDs (Sadi et al. 2018a). Our results suggested that the excellent performance of the DDCT-LEDs could be particularly useful in improving the efficiency of double diode structures (DDSs) (Sadi et al. 2018a, b) that we are using to study electroluminescent cooling (ELC) (Santhanam et al. 2013; Lee and Yen 2012; Chen et al. 2017). However, in our study Myllynen et al. (2018), we also noted that current crowding still exists in the DDCT-LEDs. Furthermore, we suggested that the current crowding could be reduced with modifying the doping levels in the structure.

In this paper we investigate the effects of modulation doping (MD) (Wang et al. 1984; Dingle et al. 1978) on a laterally doped GaInP/GaAs DDCT-LED and show that doping of the lower GaInP cladding layer and the GaAs substrate can substantially improve the current spreading and the recombination uniformity of the device. We use charge transport simulations to compare the effects of the $\mathrm{n}$ - and p-type background doping on the recombination uniformity with a doping concentration of $3 \times 10^{18} \mathrm{~cm}^{-3}$. Our results predict that the MD has an important role in the lateral current spreading in the AR of the device. In particular, the results show that p-type modulation doping can significantly improve the current spreading in the DDCT-LED with no adverse effects on device performance.

\section{Simulated structure}

Figure 1a schematically illustrates an array of lateral heterojunctions forming the DDCT-LED, and Fig. 1b shows a more detailed structure of a single DDCT-unit. The structure is based on a $\mathrm{Ga}_{0.51} \mathrm{In}_{0.49} \mathrm{P} / \mathrm{GaAs} \mathrm{DHJ}$ structure capped with a $100 \mathrm{~nm}$ thick $\mathrm{Al}_{0.3} \mathrm{Ga}_{0.7}$ As layer and a 20 $\mathrm{nm}$ thick GaAs contact layer. The laterally doped heterojunctions are formed above the AR in the GaInP and the AlGaAs layers. When the structure is forward biased, electrons and holes are electrically injected into the lateral $\mathrm{p}-\mathrm{n}$ junctions. Since the active region consists of GaAs with a band-gap energy of $1.42 \mathrm{eV}$, the required operation voltage of the LED is determined by this band gap, instead of the larger band gaps of $\mathrm{Al}_{0.3} \mathrm{Ga}_{0.7} \mathrm{As}$ and $\mathrm{Ga}_{0.51} \mathrm{In}_{0.49} \mathrm{P}$ that are $\sim 1.88$ $\mathrm{eV}$ and $\sim 1.8 \mathrm{eV}$, respectively. Consequently, the current flow through the lateral $\mathrm{p}-\mathrm{n}$ junction is negligible, and thus the charge carriers preferentially diffuse into the active region where they recombine. Furthermore, due to the injection scheme, electrons and holes are effectively 


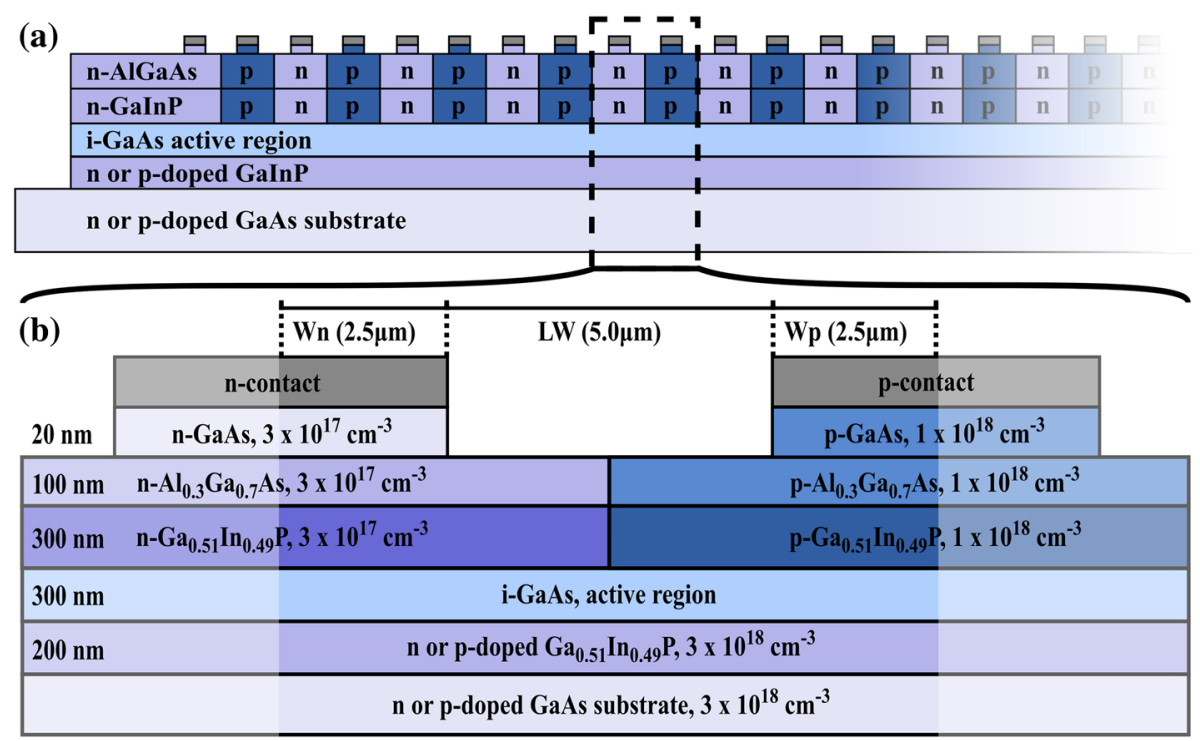

Fig. 1 a Schematic view of a DDCT-LED, and b single DDCT-unit where the simulated region extending from the middle of $\mathrm{n}$-contact to the middle of $\mathrm{p}$-contact is highlighted

separated outside the AR, essentially eliminating surface and interface recombination in the device, except at the GaInP/GaAs interfaces around the AR.

Our DDCT-LED is designed so that the LHJs can be fabricated with a selective-area doping that forms p-doped wells on the epitaxially grown n-doped layer structure. The lateral dimensions of the device are chosen so that the widths of the $n$ - and p-contacts and the uncontacted area between the contacts are equal to the fabrication linewidth (LW) of the optical lithography setup we use in ongoing experimental work. For the simulations, we have used an idealistic design for the p-doped region that is extending from the p-contact metal to the edge of the AR in the vertical direction. Also, the shape of the p-doped region is approximated with a square element for simplicity. The uncontacted thin GaAs layer between the contacts is removed with e.g. a selective wet etching process, to eliminate surface recombination at the free $\mathrm{GaAs}$ surface, and to remove a parasitic GaAs diode that would have formed within the contact layer. Our simulated device region highlighted in Fig. 1b extends from the middle of the $\mathrm{n}$-contact to the middle of the p-contact, and uses a LW of $5 \mu \mathrm{m}$.

\section{Simulation method}

Our simulations are performed at room temperature with a two-dimensional numerical transport model based on the continuity equations for charge carriers and the standard drift-diffusion currents, effectively coupling the partial differential equations for the electrostatic potential $\phi$ and the quasi-Fermi levels $E_{F p}$ and $E_{F n}$ of the valence band holes and conduction band electrons, respectively, given by

$$
\begin{gathered}
\nabla \cdot(-\epsilon \nabla \phi)=q\left(p-n+N_{d}-N_{a}\right), \\
\nabla \cdot J_{p}=\nabla \cdot\left(\mu_{p} p \nabla E_{F p}\right)=-q R,
\end{gathered}
$$




$$
\nabla \cdot J_{n}=\nabla \cdot\left(\mu_{n} n \nabla E_{F n}\right)=q R .
$$

Here $\epsilon$ is the permittivity, $q$ is the elementary charge, $p$ is the hole density in the valence band, $n$ is the electron density in the conduction band, $N_{d}$ is the ionized donor density, and $N_{a}$ is the ionized acceptor density. Also, $J_{p}\left(J_{n}\right)$ is the hole (electron) current density, $\mu_{p}\left(\mu_{n}\right)$ is the hole (electron) mobility, and $R$ is the net recombination rate per unit volume. More detailed description of the model can be found in Kivisaari et al. (2015), Kivisaari et al. (2017b), Sadi et al. (2014). The recombination rate $R$, consisting of Shockley-Read-Hall (SRH) $R_{S R H}$, radiative $R_{\text {rad }}$, and Auger recombination $R_{A u g}$, is modeled using the wellknown parameterized formula given by Kivisaari et al. (2015)

$$
\begin{aligned}
R & =R_{S R H}+R_{\text {rad }}+R_{\text {Aug }} \\
& =\left[\frac{A}{n+p+2 n_{i}}+B+C(n+p)\right]\left(n p-n_{i}^{2}\right),
\end{aligned}
$$

where $n_{i}$ is the intrinsic carrier density, and $A, B$ and $C$ are recombination constants for $\mathrm{SRH}$, radiative and Auger processes, respectively. Also, the non-radiative surface recombination rate (Chen et al. 2016) was calculated with

$$
R_{\text {surf }}=\left[\frac{V_{\text {surf }}}{n+p+2 n_{i}}\right]\left(n p-n_{i}^{2}\right),
$$

where $V_{\text {surf }}$ is the recombination velocity at the given surface. Similar equation was used for the interface recombination at the top and bottom interfaces of the AR. The recombination parameters used in our simulations are shown in Table 1.

For analyzing our results we use uniformity of the total recombination within the active region of the LED that was determined as

$$
\text { Uniformity }=\frac{\min (R)}{\max (R)},
$$

where $\min (R)$ and $\max (R)$ are the minimum and maximum rates for the total recombination, respectively. With this definition, a value of 1 corresponds to a perfect uniformity, and a value of 0 corresponds to a completely non-uniform situation. Additionally, we use the internal quantum efficiency (IQE) of the devices defined as the ratio between the radiative and the total recombination rates integrated through the device volume. Furthermore, the current density $J$ is defined as the integrated total current across the whole structure divided by the total horizontal cross-section area of the device by assuming a $100 \mu \mathrm{m}$ device length along the unsimulated axis.

Table 1 The recombination parameters used for calculating the recombination rates

\begin{tabular}{ll}
\hline Parameter & Value \\
\hline$A$ & $3 \times 10^{5}\left(\mathrm{~s}^{-1}\right)$ Bender et al. (2013) \\
$B$ & $2 \times 10^{-10}\left(\mathrm{~cm}^{3} \mathrm{~s}^{-1}\right)$ Schubert (2006) \\
$C$ & $1 \times 10^{-30}\left(\mathrm{~cm}^{6} \mathrm{~s}^{-1}\right)$ Strauss et al. (1993) \\
$V_{\text {interface }}(\mathrm{GaInP/GaAs})$ & $1.5\left(\mathrm{~cm} \mathrm{~s}^{-1}\right)$ Levinshtein et al. (1999) \\
$V_{\text {surface }}(\mathrm{AlGaAs} /$ air $)$ & $4 \times 10^{5}\left(\mathrm{~cm} \mathrm{~s}^{-1}\right)$ Levinshtein et al. (1999) \\
\hline
\end{tabular}



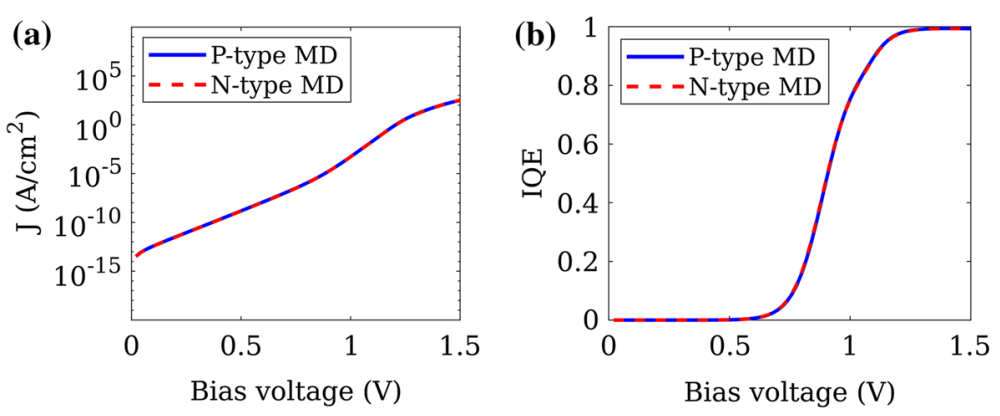

Fig. 2 a Current density and $\mathbf{b}$ internal quantum efficiency as a function of bias voltage show that device operation generally corresponds to a conventional diode and is not affected by the type of modulation doping

Fig. 3 Uniformity of total recombination for $\mathrm{n}$ - and $\mathrm{p}$ - type MD as a function of bias voltage within the active region, showing that p-type MD enables significantly higher uniformity at bias voltages above 1.1 V. Also, the uniformities corresponding to a current density of $100 \mathrm{~A} / \mathrm{cm}^{2}$ are marked with dots

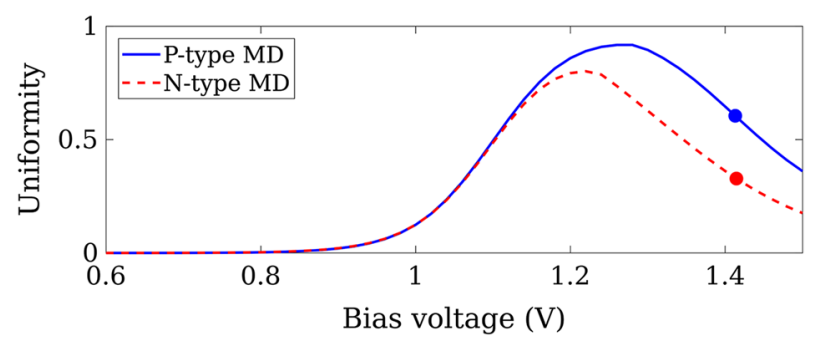

\section{Results and discussion}

In analyzing our results, we compare the performance and the current spreading of $n-$ and p-type modulation doped structures with a doping concentration of $3 \times 10^{18} \mathrm{~cm}^{-3}$. Figure 2 shows the general current-voltage and internal quantum efficiency trends of the studied structures. In Fig. 2a, a conventional diode behaviour is observed with an SRH recombination dominated region at low bias voltages, a radiative recombination dominated region at a bias range of $\sim 0.9-1.25 \mathrm{~V}$, and a resistive limited region at high biases. In Fig. $2 \mathrm{~b}$, the IQE is practically zero at SRH dominated region, after which it increases as the radiative recombination starts to increase. Most importantly, the figure shows that the changes in the background doping have no effects on the device efficiency.

Figure 3 compares the uniformity of the total recombination as a function of bias voltage within the AR of the $n$ - and p-type modulation doped LED structures. The figure shows that with p-type MD, the uniformity is significantly higher at large bias voltages above 1.1 $\mathrm{V}$, due to increased and more uniform hole concentration in the AR. Consequently, the negative effect of the imbalanced carrier mobilities, leading to current crowding, decreases. With n-type MD, the uniformity is lower, since the amount of holes available for diffusion is reduced, and the recombination becomes increasingly limited by the hole concentration. Overall, these results are in agreement with our previous study (Myllynen et al. 2018) where we observed that the recombination uniformity was generally limited by the low hole mobility.

Figure 4 shows the (a) hole and (b) electron concentrations, averaged in the vertical direction, as a function of lateral position within the AR of n- and p-type MD structures at 

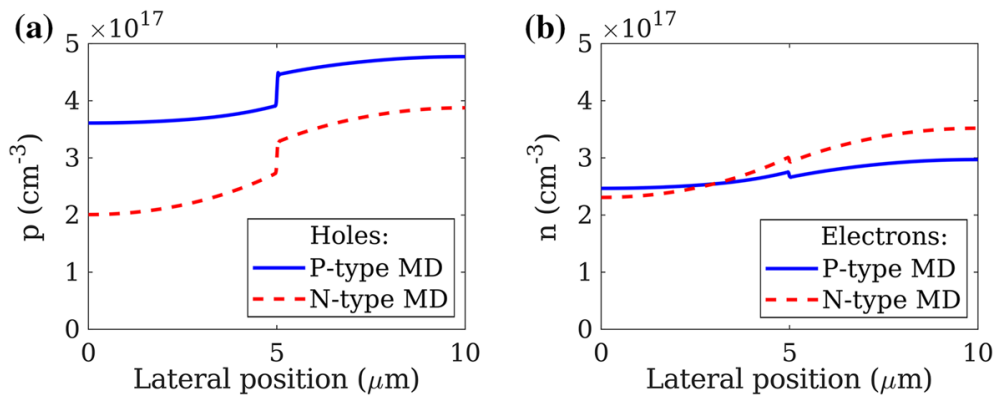

Fig. 4 Average a hole and $\mathbf{b}$ electron concentration within the AR of $n$ - and p-type MD structures as a function of lateral position at a current density of $100 \mathrm{~A} / \mathrm{cm}^{2}$

a current density of $100 \mathrm{~A} / \mathrm{cm}^{2}$. We observe that the hole concentration with p-type background doping is significantly higher than the electron concentration. As discussed earlier, this effectively overcomes the imbalanced carrier mobilities, and thus increases the recombination uniformity within the AR. In the n-type MD structure, lower uniformity is caused generally by the lack of holes under the n-doped region. In both cases, the sudden change in the doping concentrations at the middle of the AR is likely related to the carrier injection and a MD like effective doping originating from the lateral $p-n$ junction above the AR. Further analysis of the carrier concentrations suggests that most of the additional MD charge is located in a narrow channel close to the GaInP/GaAs interfaces.

Figure 5 illustrates the recombination rate within the LED structures for the n- and p-type modulation doping at a current density of $100 \mathrm{~A} / \mathrm{cm}^{2}$. In both structures, the recombination is more strongly focused under the p-contact, but with the p-type MD in Fig. 5a the difference between the maximum rate under the p-contact and the minimum rate under the n-contact is noticeably smaller compared to the n-type MD structure in Fig. 5b. Also, we observe that the recombination is evenly spread in the vertical direction despite the single-sided injection. Additionally, the recombination is fully confined within the AR, between the GaInP barriers. Overall, our results show that the modulation doping has a clear effect on the current spreading within the AR of the DDCT-LED. Furthermore, it is expected that the recombination uniformity can be further improved by optimizing the

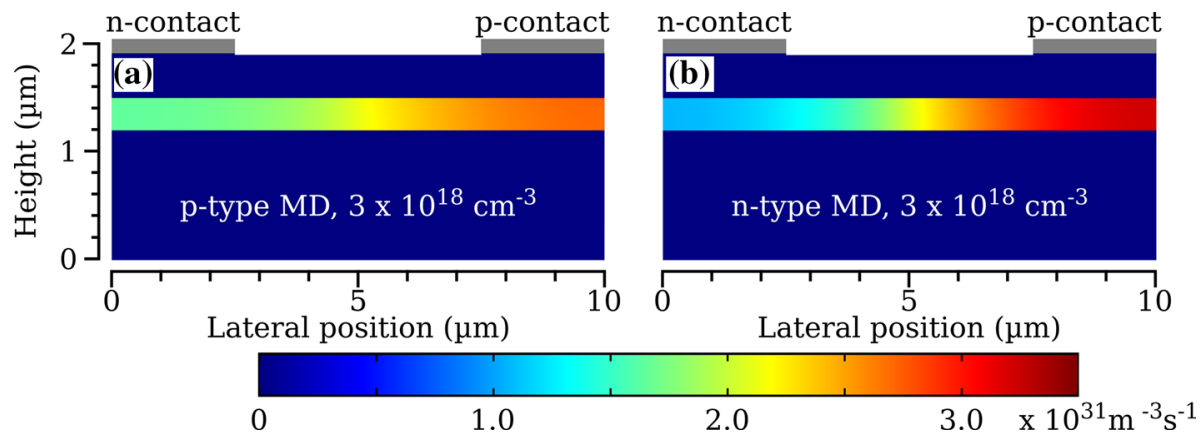

Fig. 5 Recombination rate within the a p-type and $\mathbf{b}$ n-type modulation doped structures at an average current density of $100 \mathrm{~A} / \mathrm{cm}^{2}$, showing that the recombination in p-doped LED is significantly more uniform compared to n-doped device where recombination is mostly focused under the p-contact 
lateral dimensions of the device, and by minimizing the valence band offset at the p-GaInP/ GaAs interface above AR. In a fully optimized structure, the requirements of the optical lithography process and its minimum linewidth could be lowered to allow simpler fabrication of the LEDs.

\section{Conclusions}

In conclusion, we used numerical charge transport simulations of laterally doped GaInP/ GaAs DDCT-LED to study the current spreading and recombination uniformity within the AR of the device, comparing the $n$ - and p-type background doping with doping level of $3 \times 10^{18} \mathrm{~cm}^{-3}$. We showed that modulation doping from the lower GaInP cladding layer has a strong influence on the current spreading within the AR allowing a promising route to further optimize the current spreading. Our results suggested that p-type background doping provides significantly higher recombination uniformity at high bias voltages due to the MD that increases both the spreading and the concentration of holes within the AR. Furthermore, we showed that the improved current spreading is achieved while maintaining excellent device performance.

Acknowledgements Open access funding provided by Aalto University. We acknowledge funding from the Academy of Finland and the European Research Council under the Horizon 2020 research and innovation programme (Grant Agreement No. 638173).

Open Access This article is distributed under the terms of the Creative Commons Attribution 4.0 International License (http://creativecommons.org/licenses/by/4.0/), which permits unrestricted use, distribution, and reproduction in any medium, provided you give appropriate credit to the original author(s) and the source, provide a link to the Creative Commons license, and indicate if changes were made.

\section{References}

Bender, D.A., Cederberg, J.G., Wang, C., Sheik-Bahae, M.: Development of high quantum efficiency GaAs/ GaInP double heterostructures for laser cooling. Appl. Phys. Lett. 102, 252102 (2013)

Chen, Y., Kivisaari, P., Pistol, M.-E., Anttu, N.: Optimization of the short-circuit current in an InP nanowire array solar cell through opto-electronic modeling. Nanotechnology 27, 435404 (2016)

Chen, K., Xiao, T.P., Santhanam, P., Yablonovitch, E., Fan, S.: High-performance near-field electroluminescent refrigeration device consisting of a GaAs light emitting diode and a Si photovoltaic cell. J. Appl. Phys. 122, 143104 (2017)

Dingle, R., Störmer, H., Gossard, A., Wiegmann, W.: Electron mobilities in modulation-doped semiconductor heterojunction superlattices. Appl. Phys. Lett. 33, 665-667 (1978)

Kivisaari, P., Oksanen, J., Tulkki, J.: Current injection to free-standing III-N nanowires by bipolar diffusion. Appl. Phys. Lett. 103, 031103 (2013)

Kivisaari, P., Oksanen, J., Tulkki, J., Sadi, T.: Monte Carlo simulation of hot carrier transport in III-N LEDs. J. Comput. Electron. 14, 382-397 (2015)

Kivisaari, P., Kim, I., Suihkonen, S., Oksanen, J.: Elimination of resistive losses in large-area LEDs by new diffusion-driven devices. SPIE Proc. 10124, $101240 Z$ (2017a)

Kivisaari, P., Sadi, T., Li, J., Rinke, P., Oksanen, J.: On the Monte Carlo description of hot carrier effects and device characteristics of III-N LEDs. Adv. Electron. Mater. 3, 1600494 (2017b)

Kivisaari, P., Kim, I., Suihkonen, S., Oksanen, J.: Elimination of lateral resistance and current crowding in large-area LEDs by composition grading and diffusion-driven charge transport. Adv. Electron. Mat. 3, 1700103 (2017c) 
Krames, M.R., Shchekin, O.B., Mueller-Mach, R., Mueller, G.O., Zhou, L., Harbers, G., Craford, M.G.: Status and future of high-power light-emitting diodes for solid-state lighting. J. Disp. Technol. 3, 160175 (2007)

Lee, K.-C., Yen, S.-T.: Photon recycling effect on electroluminescent refrigeration. J. Appl. Phys. 111, $014511(2012)$

Levinshtein, M., Rumyantsev, S.L., Shur, M.: Handbook Series on Semiconductor Parameters: Ternary and quaternary III-V compounds. World Scientific Publishing, London (1999)

Myllynen, A., Sadi, T., Oksanen, J.: Electrically-injected III-V diodes for large-area optoelectronics. In: Proceedings of 18th International Conference on Numerical Simulation of Optoelectonic Devices (NUSOD’18), pp. 129-130 (2018)

Nakamura, S., Krames, M.R.: History of gallium-nitride-based light-emitting diodes for illumination. Proc IEEE 101, 2211-2220 (2013)

Olsson, A., Tiira, J., Partanen, M., Hakkarainen, T., Koivusalo, E., Tukiainen, A., Guina, M., Oksanen, J.: Optical energy transfer and loss mechanisms in coupled intracavity light emitters. IEEE Trans. Electron Devices 63, 3567-3573 (2016)

Riuttanen, L., Kivisaari, P., Nykänen, H., Svensk, O., Suihkonen, S., Oksanen, J., Tulkki, J., Sopanen, M.: Diffusion injected multi-quantum well light-emitting diode structure. Appl. Phys. Lett. 104, 081102 (2014)

Riuttanen, L., Kivisaari, P., Svensk, O., Oksanen, J., Suihkonen, S.: Diffusion injection in a buried multiquantum well light-emitting diode structure. IEEE Trans. Electron Devices 62, 902-908 (2015a)

Riuttanen, L., Kivisaari, P., Svensk, O., Oksanen, J., Suihkonen, S.: Electrical injection to contactless nearsurface InGaN quantum well. Appl. Phys. Lett. 107, 051106 (2015b)

Sadi, T., Kivisaari, P., Oksanen, J., Tulkki, J.: On the correlation of the auger generated hot electron emission and efficiency droop in III-N light-emitting diodes. Appl. Phys. Lett. 105, 091106 (2014)

Sadi, T., Kivisaari, P., Tiira, J., Radevici, I., Haggren, T., Oksanen, J.: Electroluminescent cooling in intracavity light emitters: modeling and experiments. Opt. Quant. Electron 50, 18 (2018a)

Sadi, T., Radevici, I., Kivisaari, P., Casado, A., Oksanen, J.: Electroluminescent cooling using double diode structures. In: Proceedings of 18th International Conference on Numerical Simulation of Optoelectonic Devices (NUSOD’18), pp. 125-126 (2018b)

Santhanam, P., Huang, D., Gray, D.J., Ram, R.J.: Electro-luminescent cooling: light emitting diodes above unity efficiency. SPIE Proc. 8638, 863807 (2013)

Schubert, E.F.: Light-Emitting Diodes. Cambridge Universe Press, Cambridge (2006)

Strauss, U., Rühle, W.W., Köhler, K.: Auger recombination in intrinsic GaAs. Appl. Phys. Lett. 62, 55-57 (1993)

Wang, W., Mendez, E., Stern, F.: High mobility hole gas and valence-band offset in modulation-doped p-AlGaAs/GaAs heterojunctions. Appl. Phys. Lett. 45, 639-641 (1984)

Xiao, T.P., Chen, K., Santhanam, P., Fan, S., Yablonovitch, E.: Electroluminescent refrigeration by ultraefficient GaAs light-emitting diodes. J. Appl. Phys. 123, 173104 (2018)

Publisher's Note Springer Nature remains neutral with regard to jurisdictional claims in published maps and institutional affiliations. 